\title{
The inert Zee model
}

\section{Robinson Longas, Dilia Portillo, Diego Restrepo and Oscar Zapata}

Instituto de Física, Universidad de Antioquia, Calle 70 No. 52-21, Medellín, Colombia

E-mail: robinson.longas@udea.edu.co, dilia.portillo@udea.edu.co, restrepo@udea.edu.co, oalberto.zapata@udea.edu.co

ABSTRACT: We study a realization of the topology of the Zee model for the generation of neutrino masses at one-loop with a minimal set of vector-like fermions. After imposing an exact $Z_{2}$ symmetry to avoid tree-level Higgs-mediated flavor changing neutral currents, one dark matter candidate is obtained from the subjacent inert doublet model, but with the presence of new co-annihilating particles. We show that the model is consistent with the constraints coming from lepton flavor violation processes, oblique parameters, dark matter and neutrino oscillation data.

Keywords: Beyond Standard Model, Dark Matter and Double Beta Decay

ARXIV EPRINT: 1511.01873 


\section{Contents}

1 Introduction 1

2 The model 2

2.1 The scalar sector 3

2.2 Yukawa interactions and the $Z_{2}$-odd fermion sector 5

2.3 Neutrino masses 5

$\begin{array}{lll}2.4 & \text { Dark matter } & 7\end{array}$

3 Constraints $\quad 8$

3.1 Electroweak precision tests 8

$3.2 \mu \rightarrow e \gamma \quad 9$

4 Numerical results and discussion $\quad 10$

5 Conclusions $\quad 14$

$\begin{array}{ll}\text { A Free parameters } & 14\end{array}$

$\begin{array}{ll}\text { B ST formulae } & 15\end{array}$

$\begin{array}{ll}\text { C Loop function in the } \mu \rightarrow e \gamma & 15\end{array}$

\section{Introduction}

Neutrino masses and dark matter (DM) represent two phenomenological pieces of evidence for physics beyond the Standard Model (SM) which are solidly supported by the experimental data. If neutrino masses arise radiatively [1-3] it may be, though, that both originate from new physics at the $\mathrm{TeV}$ scale, and they are related to each other. In this direction, models with one-loop radiative neutrino masses and viable dark matter candidates have now a complete classification given in $[4,5]$. There, the new fields are odd under a $Z_{2}$ symmetry which ensures the stability of the DM particle, while the SM particles are even. In this work, we explore a particular model where the $Z_{2}$ can be identified with the symmetry used to avoid tree-level Higgs-mediated flavor changing neutral currents (HMFCNC) in the two Higgs doublet models (THDM) [6]. More concretely, we consider the realization of the $d=5$ Weinberg operator at one-loop order $[1,2]$ with the topology labeled as T1-ii in [2] from which the Zee model [7] is the most straightforward realization. In the Zee model, the THDM-III with tree-level HMFCNC is extended with one extra SU(2)-singlet charged-scalar. The minimal realization with two Higgs doublets of opposite parity under a $Z_{2}$ symmetry to avoid tree-level HMFCNC [8], gives rise to a diagonal-zero neutrino 
mass texture which is excluded from the measurement of a non-maximal solar neutrino mixing [9].

In this work, we extend the Zee model with a minimal set of vector-like (VL) fermions, consisting in a $\mathrm{SU}(2)_{L}$-singlet and a doublet. Then, we show that a consistent model without tree-level HMFCNC can be obtained if we impose a $Z_{2}$ symmetry to generate the Inert Doublet Model (IDM) [10]. Due to the mixing of the two resulting charged fermions, the neutral fermion cannot be the lightest $Z_{2}$-odd particle, and therefore, the DM candidate is still contained in the IDM sector of the model. In our setup, the imposed $Z_{2}$ guarantees the absence of strongly constrained flavor violating processes, relating one-loop neutrino masses with dark matter through new physics at TeV scale that can be tested at the LHC.

Another example of this kind of relation arises in the well known scotogenic models. There, the SM is increased with at least two singlet [11] or triplet [12, 13] fermions and one scalar doublet which are odd under a $Z_{2}$ symmetry. In another realization, the roles are interchanged with at least two scalar singlets and one VL doublet fermion, while one additional fermion singlet is required to close the neutrino mass loop $[14,15]$. The role of the $Z_{2}$ in the scotogenic models is to forbid tree-level contributions to the neutrino masses which are generated at one-loop level. In these models, the lightest odd particle (either scalar or fermion) can be a good DM candidate. One shared feature with the model presented here is that all the new states beyond the SM are odd under the imposed $Z_{2}$. Under this assumption, and considering new fermion and scalar fields transforming as singlets, doublets or triplets of $\mathrm{SU}(2)$, a set of 35 non-equivalent models that can simultaneously account for DM and neutrino masses at one-loop was obtained in ref. [5]. ${ }^{1}$ The model presented here is cataloged there as the T1-ii-A model with $\alpha=-2$.

This paper is organized as follows. In section 2, we present the model with its particle content and calculate the neutrino masses. Then, we analyze the DM phenomenology and establish the requirements over the free parameters necessary to reproduce the IDM phenomenology. In section 3 , we study the constraints coming from oblique parameters and present the expression for the rate of the $\mu \rightarrow e \gamma$ process. In section 4 , we present the numerical results and discuss the collider limits on VL fermions. Finally, the conclusions are presented in section 5. In the appendices, we collect the loop functions for the calculation of the oblique parameters and the $\mu \rightarrow e \gamma$ process.

\section{The model}

We start as in [7] by extending the SM with a second Higgs doublet, $H_{2}$, and a charged $\mathrm{SU}(2)$-singlet, $S^{+}$. Within this setup, Majorana neutrino masses are generated at one-loop. In this way, the Zee model is realized in the context of the general THDM-III with tree-level HMFCNC. In the model, ten new couplings are directly related to the neutrino sector. In particular, the analysis in terms of THDM-III basis independent parameters [17] was done in [18], with further analysis in [19, 20].

To avoid HMFCNC at tree-level, in the Zee-Wolfenstein model [8] was proposed the usual $Z_{2}$ symmetry in which the two doublets have opposite parity, like in Type-I or Type-

\footnotetext{
${ }^{1} \mathrm{~A}$ comprehensive list of the radiative seesaw literature is given in [16].
} 


\begin{tabular}{|c|c|c|}
\hline & Spin & $\mathrm{SU}(3)_{C}, \mathrm{SU}(2)_{L}, \mathrm{U}(1)_{Y}, Z_{2}$ \\
\hline$\epsilon$ & $1 / 2$ & $(\mathbf{1}, \mathbf{1},-2,-)$ \\
\hline$\Psi$ & $1 / 2$ & $(\mathbf{1}, \mathbf{2},-1,-)$ \\
\hline$H_{2}$ & 0 & $(\mathbf{1}, \mathbf{2}, 1,-)$ \\
\hline$S^{-}$ & 0 & $(\mathbf{1}, \mathbf{1},-2,-)$ \\
\hline
\end{tabular}

Table 1. The new particle content of the model with their transformation properties under the $\mathrm{SU}(3)_{C} \otimes \mathrm{SU}(2)_{L} \otimes \mathrm{U}(1)_{Y} \otimes Z_{2}$ symmetry.

II or other THDM realizations [6]. Under this symmetry, the Lagrangian relevant for the neutrino mass generation requires $S^{ \pm}$to be $Z_{2}$-even, and hence a $Z_{2}$ soft-breaking mass parameter needs to be introduced in the scalar sector, which, in joint with the three antisymmetric Yukawa couplings of $S^{ \pm}$with the lepton doublets of different families, account for only four new couplings directly related to the neutrino sector. This minimal model, however, turns to be not enough to fit the observables related to neutrino oscillation data and is now excluded [9].

In this work, we want to explore the minimal realization of the T1-ii topology of [2], which is safe regarding strongly constrained lepton-flavor violation, in particular, without tree-level HMFCNC. We start by assigning a $Z_{2}$-odd charge to both $S^{ \pm}$and the second Higgs doublet $H_{2}$. At this level, the resulting model would be a Type-I THDM with an extra $S^{ \pm}$and massless neutrinos. After that, we propose one minimal extension of this setup that only involves six additional Yukawa-couplings related to neutrino physics (instead of the nine of the general Zee model without the $Z_{2}$ ). This consists of adding a $Z_{2}$-odd pair

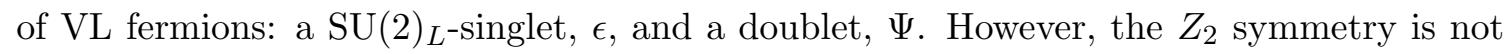
enough to avoid mixing of the new VL fermions with the SM leptons which could regenerate tree-level HMFCNC, as well as other lepton flavor violating processes subject to several (stringent) constraints [21-25]. Therefore, we impose in addition that the neutral part of $\mathrm{H}_{2}$ does not develop a vacuum expectation value (vev). In this way, the IDM is obtained, which includes a potential scalar DM candidate. To our knowledge, the model was first proposed in the catalog of the realization of the $d=5$ Weinberg operator at one-loop with DM candidates [5] and labeled there as T1-ii-A model with $\alpha=-2$.

The new particle content and their charges are summarized in the table 1. A similar approach with controlled FCNC and DM was followed in [26] where the minimal supersymmetric standard model was extended with two $\mathrm{SU}(2)$-singlet opposite-charge superfields.

\subsection{The scalar sector}

The most general $Z_{2}$-invariant scalar potential of the model is given by

$$
\begin{aligned}
V= & \mu_{1}^{2} H_{1}^{\dagger} H_{1}+\mu_{2}^{2} H_{2}^{\dagger} H_{2}+\frac{\lambda_{1}}{2}\left(H_{1}^{\dagger} H_{1}\right)^{2}+\frac{\lambda_{2}}{2}\left(H_{2}^{\dagger} H_{2}\right)^{2}+\lambda_{3}\left(H_{1}^{\dagger} H_{1}\right)\left(H_{2}^{\dagger} H_{2}\right) \\
& +\lambda_{4}\left(H_{1}^{\dagger} H_{2}\right)\left(H_{2}^{\dagger} H_{1}\right)+\frac{\lambda_{5}}{2}\left[\left(H_{1}^{\dagger} H_{2}\right)^{2}+\text { h.c. }\right]+\mu_{S}^{2} S^{+} S^{-} \\
& +\lambda_{S}\left(S^{+} S^{-}\right)^{2}+\lambda_{6}\left(S^{+} S^{-}\right) H_{1}^{\dagger} H_{1}+\lambda_{7}\left(S^{+} S^{-}\right)\left(H_{2}^{\dagger} H_{2}\right)+\mu \epsilon_{a b}\left[H_{1}^{a} H_{2}^{b} S^{-}+\text {h.c. }\right],
\end{aligned}
$$


where $\epsilon_{a b}$ is the $\mathrm{SU}(2)_{L}$ antisymmetric tensor with $\epsilon_{12}=1, H_{1}=\left(0, H_{1}^{0}\right)^{\mathrm{T}}$ is the SM Higgs doublet and $H_{2}=\left(H_{2}^{+}, H_{2}^{0}\right)^{\mathrm{T}}$. The scalar couplings $\lambda_{5}$ and $\mu$ are taken to be real. After the electroweak symmetry breaking, the neutral scalar fields can be parametrized in the form $H_{2}^{0}=\left(H^{0}+i A^{0}\right) / \sqrt{2}$ and $H_{1}^{0}=(h+v) / \sqrt{2}$, with $h$ being the Higgs boson and $v=246 \mathrm{GeV}$. Note that $H_{2}^{0}$ does not develop a vacuum expectation value in order to ensure the conservation of the $Z_{2}$ symmetry. The neutral scalar spectrum coincides with the one of the IDM $[10,27,28]$, which consists of two CP-even neutral states $\left(H^{0}, h\right)$ and a CP-odd neutral state $\left(A^{0}\right)$. The masses of the $Z_{2}$-odd neutral scalar particles read as

$$
m_{H^{0}}^{2}=\mu_{2}^{2}+\frac{1}{2}\left(\lambda_{3}+\lambda_{4}+\lambda_{5}\right) v^{2}, \quad m_{A^{0}}^{2}=\mu_{2}^{2}+\frac{1}{2}\left(\lambda_{3}+\lambda_{4}-\lambda_{5}\right) v^{2} .
$$

On the other hand, the charged scalar sector involves a mixture of the singlet and doublet $Z_{2}$-odd charged states which leads to the following mass matrix in the basis $\left(H_{2}^{ \pm}, S^{ \pm}\right)$

$$
\mathcal{M}_{S}^{2}=\left(\begin{array}{cc}
m_{H^{ \pm}}^{2} & \frac{-\mu v}{\sqrt{2}} \\
\frac{-\mu v}{\sqrt{2}} & m_{S^{ \pm}}^{2}
\end{array}\right)
$$

where $m_{H^{ \pm}}^{2}=\mu_{2}^{2}+\frac{1}{2} \lambda_{3} v^{2}$ and $m_{S^{ \pm}}^{2}=\mu_{S}^{2}+\frac{1}{2} \lambda_{6} v^{2}$. The mass eigenstates $\kappa_{1}^{ \pm}$and $\kappa_{2}^{ \pm}$are defined as

$$
\left(\begin{array}{c}
H_{2}^{ \pm} \\
S^{ \pm}
\end{array}\right)=\left(\begin{array}{cc}
\cos \delta & -\sin \delta \\
\sin \delta & \cos \delta
\end{array}\right)\left(\begin{array}{c}
\kappa_{1}^{ \pm} \\
\kappa_{2}^{ \pm}
\end{array}\right), \quad \sin 2 \delta=\frac{\sqrt{2} \mu v}{m_{\kappa_{2}^{+}}^{2}-m_{\kappa_{1}^{+}}^{2}}
$$

with the corresponding masses

$$
m_{\kappa_{1,2}^{ \pm}}^{2}=\frac{1}{2}\left(m_{H^{ \pm}}^{2}+m_{S^{ \pm}}^{2} \mp \sqrt{\left(m_{H^{ \pm}}^{2}-m_{S^{ \pm}}^{2}\right)^{2}+2 \mu^{2} v^{2}}\right)
$$

with $\mu$ constrained from above by the requirement of having $m_{\kappa_{1}^{+}}^{2}>0$.

Lastly, the scalar couplings are subject to perturbativity and vacuum stability constraints, which imply the following conditions [10, 29]:

$$
\begin{aligned}
& \mu_{1}^{2}<0, \quad \lambda_{1} \mu_{2}^{2}>\lambda_{3} \mu_{1}^{2}, \quad \lambda_{1} \mu_{S}^{2}>\lambda_{6} \mu_{1}^{2}, \quad \lambda_{1} \mu_{2}^{2}>\left(\lambda_{3}+\lambda_{4} \pm\left|\lambda_{5}\right|\right) \mu_{1}^{2}, \quad\left|\lambda_{S}\right|,\left|\lambda_{i}\right|<8 \pi, \\
& \lambda_{1}, \lambda_{2}, \quad \lambda_{S}>0, \quad \lambda_{6}>-\sqrt{\frac{\lambda_{1} \lambda_{S}}{2}}, \quad \lambda_{7}>-\sqrt{\frac{\lambda_{2} \lambda_{S}}{2}}, \quad \lambda_{3}+\lambda_{4}-\left|\lambda_{5}\right|+\sqrt{\lambda_{1} \lambda_{2}}>0 .
\end{aligned}
$$

These theoretical conditions constrain the mass splittings among the $Z_{2}$-odd scalar particles.

With regard to the free parameters in the scalar sector, it is possible to choose the following set

$$
m_{H^{0}}, m_{A^{0}}, m_{\kappa_{1}^{+}}, m_{\kappa_{2}^{+}}, \lambda_{L}, \lambda_{6}, \delta,
$$

where $\lambda_{L}=\frac{1}{2}\left(\lambda_{3}+\lambda_{4}+\lambda_{5}\right)$ controls the trilinear coupling between the SM Higgs and $H^{0}$. Because the quartic couplings $\lambda_{2}, \lambda_{S}$ and $\lambda_{7}$ are only relevant for interactions exclusively involving $Z_{2}$-odd particles, they can be left apart in a tree-level analysis. ${ }^{2}$ The

\footnotetext{
${ }^{2}$ Note that at one-loop level $\lambda_{2}$ and $\lambda_{7}$ may play a main role in processes such as the DM annihilations into $\gamma \gamma$ and $Z \gamma$ [30,31], DM scattering on nucleons [32] and other radiative processes [33].
} 
relation between the remaining scalar couplings and the scalar masses are presented in the appendix A. From eqs. (2.2) and (2.5), we can expect that for appropriate scalar couplings, $H^{0}$ or $A^{0}$ can be the lightest $Z_{2}$-odd scalar particle in the scalar spectrum.

\subsection{Yukawa interactions and the $Z_{2}$-odd fermion sector}

The $Z_{2}$-invariant Lagrangian respecting the SM gauge symmetry contains the following new terms

$$
-\mathcal{L} \supset\left\{\eta_{i} \bar{L}_{i} H_{2} \epsilon+\rho_{i} \bar{\Psi} H_{2} e_{R i}+\Pi \bar{\Psi} H_{1} \epsilon+f_{i}^{*} \overline{L_{i}^{c}} \Psi S^{+}+\text {h.c }\right\}+m_{\Psi} \bar{\Psi} \Psi+m_{\epsilon} \bar{\epsilon} \epsilon,
$$

where $L_{i}$ and $e_{R i}$ are the lepton doublets and $\mathrm{SU}(2)$-singlets respectively, $\Psi=(N, E)^{\mathrm{T}}$ is the VL doublet, $\Pi, \eta_{i}, \rho_{i}$ and $f_{i}$ are Yukawa-couplings controlling the new interactions, and $i$ is the family index. As it will be shown below, the $\eta_{i}, f_{i}$ terms with the mixing terms $\Pi$ and $\mu$ give rise to nonzero neutrino masses at one loop level, and along with the $\rho_{i}$ term, induce lepton flavor violation (LFV) processes such as $\mu \rightarrow e \gamma$.

Once the electroweak symmetry is spontaneously broken the $\Pi$ term generates a mixture of the two charged $Z_{2}$-odd fermions, leading to a mass matrix in the basis $(E, \epsilon)$ given by $^{3}$

$$
\mathcal{M}=\left(\begin{array}{cc}
m_{\Psi} & \frac{\Pi v}{\sqrt{2}} \\
\frac{\Pi v}{\sqrt{2}} & m_{\epsilon}
\end{array}\right)
$$

The charged mass eigenstates $\chi_{1}$ and $\chi_{2}$ are defined by

$$
\left(\begin{array}{c}
E \\
\epsilon
\end{array}\right)=\left(\begin{array}{cc}
\cos \alpha & -\sin \alpha \\
\sin \alpha & \cos \alpha
\end{array}\right)\left(\begin{array}{l}
\chi_{1} \\
\chi_{2}
\end{array}\right), \quad \sin 2 \alpha=\frac{\sqrt{2} \Pi v}{m_{\chi_{2}}-m_{\chi_{1}}},
$$

with masses

$$
m_{\chi_{1,2}}=\frac{1}{2}\left(m_{\Psi}+m_{\epsilon} \mp \sqrt{\left(m_{\Psi}-m_{\epsilon}\right)^{2}+2 \Pi^{2} v^{2}}\right) .
$$

The $Z_{2}$-odd fermion spectrum also contains a neutral Dirac fermion $N$, with a mass $m_{N}=$ $m_{\Psi}$. From above expression, it follows that $m_{N}=m_{\chi_{1}} \cos ^{2} \alpha+m_{\chi_{2}} \sin ^{2} \alpha$, which implies the hierarchical spectrum $m_{\chi_{1}} \leq m_{N} \leq m_{\chi_{2}}$. In other words, the neutral fermion $N$ can not be the lightest $Z_{2}$-odd particle in the spectrum.

\subsection{Neutrino masses}

The usual lepton number $(L)$ assignment in the Zee model corresponds to $L\left(H_{2}\right)=0$ and $L(S)=-2$, which makes the $\mu$ term in the scalar potential the only explicit $L$-violating term in the Lagrangian. Hence, by keeping such assignment and charging under $L$ the new fermion fields as $L(\Psi)=L(\epsilon)=+1$, in order to make the Yukawa interactions $L$ conserving, the $\mu$ term is again the responsible for the $L$ breaking in the model, and the subsequent neutrino Majorana masses and lepton flavor violation processes.

\footnotetext{
${ }^{3}$ For simplicity we have assumed $\Pi$ to be real.
} 

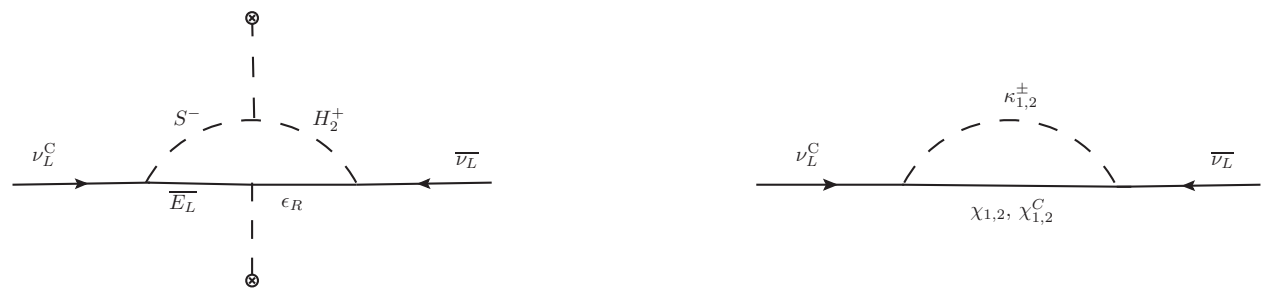

Figure 1. One-loop diagram for neutrino masses in the interaction (left-panel) and mass (rightpanel) basis.

Non-zero neutrino masses at one-loop are generated in this model thanks to the combination of the Yukawa-coupling $\eta_{i}$ and $f_{i}$, the scalar mixing $\mu$, and fermion mixing $\Pi$, as displayed in the left-panel of figure 1. The corresponding Majorana mass-matrix in the mass-eigenstate basis, calculated from the Feynman diagram displayed in the right-panel of figure 1 , takes the form

$$
\left[M^{\nu}\right]_{i j}=\frac{\sin 2 \alpha \sin 2 \delta}{64 \pi^{2}}\left(\eta_{i} f_{j}+\eta_{j} f_{i}\right) \sum_{n} c_{n} m_{\chi_{n}} I\left(m_{\kappa_{1}^{+}}^{2}, m_{\kappa_{2}^{+}}^{2}, m_{\chi_{n}}^{2}\right) .
$$

Here $c_{1}=-1, c_{2}=+1$ and the loop function is given by

$$
I\left(m_{a}^{2}, m_{b}^{2}, m_{c}^{2}\right)=\frac{m_{b}^{2}}{m_{b}^{2}-m_{c}^{2}} \ln \left(\frac{m_{b}^{2}}{m_{c}^{2}}\right)-\frac{m_{a}^{2}}{m_{a}^{2}-m_{c}^{2}} \ln \left(\frac{m_{a}^{2}}{m_{c}^{2}}\right) .
$$

Due to the flavor structure of $M^{\nu}$, it has a zero determinant and, therefore, contains only two massive neutrinos. In this way, the number of Majorana phases is reduced to only one, and neutrinos masses are entirely set by the solar and atmospheric mass differences. Specifically, for normal hierarchy $(\mathrm{NH}) m_{1}=0, m_{2}=\sqrt{\Delta m_{\mathrm{sol}}^{2}}$ and $m_{3}=\sqrt{\Delta m_{\text {atm }}^{2}}$ while for inverted hierarchy (IH) $m_{1}=\sqrt{\Delta m_{\mathrm{atm}}^{2}}, m_{2}=\sqrt{\Delta m_{\mathrm{sol}}^{2}+m_{1}^{2}} \approx \sqrt{\Delta m_{\mathrm{atm}}^{2}}$ and $m_{3}=0$. On the other hand, $M^{\nu}$ depends on the scalar and fermion mixing angles with vanisihing entries for either $m_{\kappa_{1}^{+}}=m_{\kappa_{2}^{+}}$, or $m_{\chi_{1}}=m_{\chi_{2}}$. Thus, to have small neutrino masses a degenerate mass spectrum up to some extent could be required. By taking the trace of $M^{\nu}$ we can estimate the values of the different quantities involved in the calculation of neutrino masses:

$$
\begin{aligned}
\operatorname{Tr}\left[M^{\nu}\right] \approx \sqrt{\Delta m_{\text {atm }}^{2}}=0.03 \mathrm{eV} & \left(\frac{\sin 2 \alpha \sin 2 \delta}{10^{-2}}\right)\left(\frac{|\vec{\eta} \cdot \vec{f}|}{10^{-6}}\right) \times \\
& \times \sum_{n} c_{n}\left(\frac{m_{\chi_{n}}}{100 \mathrm{GeV}}\right) I\left(m_{\kappa_{1}^{+}}^{2}, m_{\kappa_{2}^{+}}^{2}, m_{\chi_{n}}^{2}\right) .
\end{aligned}
$$

This means that barring cancellations in the mass sector, and between Yukawa-couplings, small mixing angles and Yukawa-couplings are required. Certainly large values for the Yukawa-couplings can be obtained for smaller values of $\sin 2 \alpha \sin 2 \delta$ or more compressed mass spectra.

The neutrino mass matrix is diagonalized by the Pontecorvo-Maki-Nakagawa-Sakata mixing matrix $U_{\mathrm{PMNS}}[34]$ as

$$
U_{\mathrm{PMNS}}^{\mathrm{T}} M^{\nu} U_{\mathrm{PMNS}}=\operatorname{diag}\left(m_{1}, m_{2}, m_{3}\right), \quad m_{i} \geq 0,
$$


which can be written in the form $U_{\mathrm{PMNS}}=V P$ [35], where the matrix $V$ contains the neutrino mixing angles and the $\mathrm{CP}$ Dirac phase and $P=\operatorname{diag}\left(1, e^{i \alpha / 2}, 1\right)$ carries the dependence on the CP Majorana phase. It is worth mentioning that for $\alpha=0, \pm \pi, \pm 2 \pi$, the Majorana phase does not contribute to the $\mathrm{CP}$ violation and in such a case the relative $\mathrm{CP}$ parity of the two massive neutrinos would be $\lambda=e^{ \pm i \alpha}= \pm 1$. From eq. (2.15) and thanks to the flavor structure of the neutrino mass matrix, given by eq. (2.12), we can express five of the six Yukawa-couplings $\eta_{i}$ and $f_{i}$ in terms of the neutrino observables. Without loss of generality $\eta_{1}$ can be chosen to be the free parameter which can be restricted using other low energy observables such as $\mu \rightarrow e \gamma$. Thus, the most general Yuwawa-couplings that are compatible with the neutrino oscillation data are given by

$$
\vec{\eta}=\left|\eta_{1}\right|\left(\begin{array}{c}
e^{i \phi_{\eta_{1}}} \\
A_{2} / \beta_{11} \\
A_{3} / \beta_{11}
\end{array}\right), \vec{f}=\frac{1}{2 \zeta}\left(\begin{array}{c}
\beta_{11} / \eta_{1} \\
\beta_{22} / \eta_{2} \\
\beta_{33} / \eta_{3}
\end{array}\right)=\frac{\beta_{11}}{2 \zeta\left|\eta_{1}\right|}\left(\begin{array}{c}
e^{i \phi_{\eta_{1}}} \\
\beta_{22} / A_{2} \\
\beta_{33} / A_{3}
\end{array}\right)
$$

where we have defined

$$
\begin{aligned}
\beta_{i j} & =\lambda m_{2} V_{i 2}^{*} V_{j 2}^{*}+m_{3} V_{i 3}^{*} V_{j 3}^{*}, \\
A_{j} & = \pm \sqrt{-\lambda m_{2} m_{3}\left(V_{12}^{*} V_{j 3}^{*}-V_{13}^{*} V_{j 2}^{*}\right)^{2}}+\beta_{1 j} e^{i \operatorname{Arg}\left(\eta_{1}\right)}, \text { for } \mathrm{NH}, \\
\beta_{i j} & =m_{1} V_{i 1}^{*} V_{j 1}^{*}+\lambda m_{2} V_{i 2}^{*} V_{j 2}^{*}, \\
A_{j} & = \pm \sqrt{-\lambda m_{1} m_{2}\left(V_{11}^{*} V_{j 2}^{*}-V_{12}^{*} V_{j 1}^{*}\right)^{2}}+\beta_{1 j} e^{i \operatorname{Arg}\left(\eta_{1}\right)}, \text { for } \mathrm{IH} \\
\zeta & =\frac{\sin 2 \alpha \sin 2 \delta}{64 \pi^{2}} \sum_{n} c_{n} m_{\chi_{n}} I\left(m_{\kappa_{1}^{+}}^{2}, m_{\kappa_{2}^{+}}^{2}, m_{\chi_{n}}^{2}\right) .
\end{aligned}
$$

In this way, it is always possible to correctly reproduce the neutrino oscillation parameters in the present model. Note that, in general, the non-free Yukawa-couplings are complex numbers. However, they become real in a CP-conserving scenario with $\lambda=-1$ and $\eta_{1}$ being real.

\subsection{Dark matter}

The $Z_{2}$ symmetry renders the lightest $Z_{2}$-odd particle stable, and if it is electrically neutral then it can play the role of the DM particle. Since $m_{\chi_{1}} \leq m_{N}$, doublet fermion DM can not take place in this model. ${ }^{4}$ Therefore, only the neutral $Z_{2}$-odd scalars, either $H^{0}$ or $A^{0}$, can be the DM candidates. This makes this model to resemble up to some extent the IDM from the DM phenomenology point of view. Accordingly, two possible scenarios emerge depending on whether the particles not belonging to the $\operatorname{IDM}\left(S^{ \pm}, \chi_{1,2}\right.$ and $\left.N\right)$ participate or not in the DM annihilation. When these particles do take part of DM annihilation, the extra (not present in the IDM) coannihilation processes are the ones mediated by the Yukawa-couplings $\eta_{i}, f_{i}$ and $\rho_{i}$, and by the scalar couplings $\mu$ and $\lambda_{6}$.

\footnotetext{
${ }^{4}$ Furthermore, since $N$ has a direct coupling to the $Z$ gauge boson which gives rise to a spin-independent cross section orders of magnitude larger than present limits, it is excluded as a viable DM candidate.
} 
For the scenario without the extra coannihilation processes, the DM phenomenology is expected to be similar to that of the IDM by assuming $m_{\kappa_{2}^{+}}, m_{\chi_{1}} \gg m_{\kappa_{1}^{+}}$, a small scalar mixing angle and $\eta_{i}, f_{i}, \rho_{i}, \lambda_{6} \ll 1$. In addition, $\mu / v=\sin 2 \delta\left(m_{\kappa_{2}^{+}}^{2}-m_{\kappa_{1}^{+}}^{2}\right) / \sqrt{2} v^{2} \ll 1$ must also be satisfied. In this way, the coannihilation effects of the mentioned particles with the DM particle can be neglected. Note that the requirement of having small Yukawacouplings is also in agreement with neutrino masses and $\mu \rightarrow e \gamma$ as it will be shown below. It follows that the viable DM mass range for this scenario (the same of the one in the IDM) is composed by two regions $[27,28,33,36-39]:^{5}$ the low mass regime, $m_{H^{0}} \simeq m_{h} / 2$, and the high mass regime, $m_{H^{0}} \gtrsim 500 \mathrm{GeV}$. In the region $100 \mathrm{GeV} \lesssim m_{H^{0}}<500 \mathrm{GeV}$ the gauge interactions become large so that it is not possible to reach the observed relic density, i.e. $\Omega_{H^{0}}<\Omega_{D M}$. In the Higgs funnel region, DM self-annihilations through the Higgs $s$ channel exchange provide the dominant contribution to the DM annihilation cross section, with $\lambda_{L}$ and $m_{H^{0}}$ as the relevant parameters. LEP measurements give rise to the following constraints: $m_{H^{0}}+m_{A^{0}}>M_{Z}, \max \left(m_{H^{0}}, m_{A^{0}}\right)>100 \mathrm{GeV}$ and $m_{\kappa_{1}^{+}} \gtrsim 70 \mathrm{GeV}$. On the other hand, for DM masses larger than $500 \mathrm{GeV}$ the relic abundance strongly depends on the mass splittings between $H^{0}, A^{0}$ and $\kappa_{1}^{ \pm}$. Indeed, a small splitting of at most $15 \mathrm{GeV}$ is required to reproduce the correct relic density implying that coannihilations between those particles must be taken into account.

Regarding the scenario where $S^{ \pm}, \chi_{1,2}$ and $N$ contribute to the DM annihilation, the extra coannihilation processes involve the following initial states: $H_{2}^{0} \chi_{i}, H_{2}^{0} \kappa_{i}^{ \pm}, \chi_{i} \kappa_{j}^{ \pm}$, $N \kappa_{j}^{ \pm}, \kappa_{i}^{ \pm} \kappa_{j}^{ \pm}$. These processes might play the main role in the calculation of the DM relic density affecting in a sensible way the expectations for DM detection [40-42] and, therefore, modifying the viable parameter space of the model. Since a detailed analysis of the impact of these extra coannihilation channels on the relic density is beyond the scope of this work, in what follows we will no longer consider this scenario.

\section{Constraints}

\subsection{Electroweak precision tests}

In the present model, the new fields may modify the vacuum polarization of gauge bosons whose effects are parametrized by the $S, T$ and $U$ electroweak parameters [43]. The new fermion $\left(S_{F}, T_{F}\right)$ and scalar $\left(S_{S}, T_{S}\right)$ contributions to the $S$ and $T$ parameters are [44-46]: ${ }^{6}$

$$
\begin{aligned}
S_{F}=\frac{1}{3 \pi}\left[2 s_{\alpha}^{2} c_{\alpha}^{2}\right. & {\left.\left[1-3 \Theta_{S}\left(m_{\chi_{1}}^{2}, m_{\chi_{2}}^{2}\right)\right]+\log \left(\frac{m_{\chi_{2}}^{2}}{m_{N}^{2}}\right)+c_{\alpha}^{2} \log \left(\frac{m_{\chi_{1}}^{2}}{m_{\chi_{2}}^{2}}\right)\right] } \\
T_{F}=\frac{1}{4 \pi m_{W}^{2} s_{W}^{2}} & {\left[\frac{\left(m_{\chi_{1}}-m_{\chi_{2}}\right)^{2}}{2}\left[2 c_{\alpha}^{4} \log \left(\frac{m_{\chi_{2}}^{2}}{m_{N} m_{\chi_{1}}}\right)+c_{\alpha}^{2} \log \left(\frac{m_{N}^{2}}{m_{\chi_{2}}^{2}}\right)+c_{\alpha}^{6} \log \left(\frac{m_{\chi_{1}}^{2}}{m_{\chi_{2}}^{2}}\right)\right]\right.} \\
+ & \left.2 c_{\alpha}^{2} \Theta_{T}\left(m_{\chi_{1}}^{2}, m_{N}^{2}\right)+2 s_{\alpha}^{2} \Theta_{T}\left(m_{\chi_{2}}^{2}, m_{N}^{2}\right)-2 s_{\alpha}^{2} c_{\alpha}^{2} \Theta_{T}\left(m_{\chi_{1}}^{2}, m_{\chi_{2}}^{2}\right)\right]
\end{aligned}
$$

\footnotetext{
${ }^{5}$ Without loss of generality we assume $H^{0}$ to be the DM candidate.

${ }^{6}$ Because the $U$ parameter is suppressed by the new physics scale $U \sim\left(M_{W} / \Lambda\right)^{2} T$, we do not take it into account [47].
} 


$$
\begin{aligned}
& S_{S}=\frac{1}{4 \pi m_{Z}^{2}}\left[c_{\delta}^{2}\left(c_{\delta}^{2}-2\right) \Theta\left(m_{Z}^{2} ; m_{\kappa_{1}^{+}}, m_{\kappa_{1}^{+}}\right)+\Theta\left(m_{Z}^{2} ; m_{H^{0}}, m_{A^{0}}\right)\right. \\
&-\Theta\left(0 ; m_{H^{0}}, m_{A^{0}}\right)+s_{\delta}^{2}\left(s_{\delta}^{2}-2\right) \Theta\left(m_{Z}^{2} ; m_{\kappa_{2}^{+}}, m_{\kappa_{2}^{+}}\right) \\
&\left.+2 s_{\delta}^{2} c_{\delta}^{2}\left[\Theta\left(m_{Z}^{2} ; m_{\kappa_{1}^{+}}, m_{\kappa_{2}^{+}}\right)-\Theta\left(0 ; m_{\kappa_{1}^{+}}, m_{\kappa_{2}^{+}}\right)\right]\right], \\
& T_{S}=\frac{1}{16 \pi m_{W}^{2} s_{W}^{2}}\left[c_{\delta}^{2} \Theta\left(0 ; m_{\kappa_{1}^{+}}, m_{H^{0}}\right)+c_{\delta}^{2} \Theta\left(0 ; m_{\kappa_{1}^{+}}, m_{A^{0}}\right)+s_{\delta}^{2} \Theta\left(0 ; m_{\kappa_{2}^{+}}, m_{H^{0}}\right)\right. \\
&\left.\quad+s_{\delta}^{2} \Theta\left(0 ; m_{\kappa_{2}^{+}}, m_{A^{0}}\right)-\Theta\left(0 ; m_{A^{0}}, m_{H^{0}}\right)-2 s_{\delta}^{2} c_{\delta}^{2} \Theta\left(0 ; m_{\kappa_{1}^{+}}, m_{\kappa_{2}^{+}}\right)\right],
\end{aligned}
$$

where $c_{\alpha}=\cos \alpha, s_{\alpha}=\sin \alpha, c_{\delta}=\cos \delta, s_{\delta}=\sin \delta$ and the loop functions $\Theta$ are given in the appendix B. From these expressions we can see that the fermion contributions to $T_{F}$ and $S_{F}$ vanish in the limiting case of $\alpha=0$, which points out to the existence of a custodial symmetry. For that reason we do not expect large deviations on $S$ and $T$ for a small mixing angle $\alpha$. In contrast, the scalar contributions do not tend to zero for $\delta=0$ due to the fact that after the electroweak symmetry breaking the components of the $Z_{2}$-odd doublet $H_{2}$ have mass splittings that are independent of $\delta$. However, the agreement with electroweak precision tests is reached due to the small mass splitting between $A^{0}$ and $\kappa_{1}^{ \pm}\left(H^{0}, A^{0}\right.$ and $\kappa_{1}^{ \pm}$) in the low (high) mass regime, just as it happens in the IDM.

\section{$3.2 \mu \rightarrow e \gamma$}

Lepton flavor violation processes could be a clear signal of new physics. However, due to the lack of any signal in this sector, very stringent constraints over the branching ratios for particular processes are set, with $\mu \rightarrow e \gamma$ being one of the most constraining processes. In this model such a process is controlled by the $\eta_{1,2}, f_{1,2}$ and $\rho_{1,2}$ Yukawa-couplings and mediated by the $Z_{2}$-odd particles. Certainly, the interactions in eq. (2.8) and the scalar mixing term allow to construct the one-loop diagram shown in figure 2 . The branching ratio for $\mu \rightarrow e \gamma$ process reads

$$
\mathcal{B}(\mu \rightarrow e \gamma)=\frac{3 \alpha_{e m}}{64 \pi m_{\mu}^{2} G_{F}^{2}}\left(\left|\Sigma_{L}\right|^{2}+\left|\Sigma_{R}\right|^{2}\right)
$$

where $\alpha_{e m}$ is the electromagnetic fine structure constant, $G_{F}$ is the Fermi constant and $\Sigma_{L}, \Sigma_{R}$ are given by

$$
\begin{aligned}
\Sigma_{L}= & -\rho_{1}^{*} \eta_{2}^{*} s_{\alpha} c_{\alpha}\left[m_{\chi_{1}} \mathcal{G}_{1}\left(m_{\chi_{1}}^{2}, m_{A^{0}}^{2}, m_{H^{0}}^{2}\right)-m_{\chi_{2}} \mathcal{G}_{1}\left(m_{\chi_{2}}^{2}, m_{A^{0}}^{2}, m_{H^{0}}^{2}\right)\right] \\
& -m_{\mu} \rho_{1} \rho_{2}^{*}\left[s_{\alpha}^{2} \mathcal{F}_{1}\left(m_{\chi_{2}}^{2}, m_{A^{0}}^{2}, m_{H^{0}}^{2}\right)+c_{\alpha}^{2} \mathcal{F}_{1}\left(m_{\chi_{1}}^{2}, m_{A^{0}}^{2}, m_{H^{0}}^{2}\right)\right] \\
& +m_{\mu} \rho_{1} \rho_{2}^{*}\left[c_{\delta}^{2} \mathcal{F}_{2}\left(m_{\kappa_{1}^{+}}^{2}, m_{N}^{2}\right)+s_{\delta}^{2} \mathcal{F}_{2}\left(m_{\kappa_{2}^{+}}^{2}, m_{N}^{2}\right)\right] \\
\Sigma_{R}= & -\rho_{2} \eta_{1} s_{\alpha} c_{\alpha}\left[m_{\chi_{1}} \mathcal{G}_{1}\left(m_{\chi_{1}}^{2}, m_{A^{0}}^{2}, m_{H^{0}}^{2}\right)-m_{\chi_{2}} \mathcal{G}_{1}\left(m_{\chi_{2}}^{2}, m_{A^{0}}^{2}, m_{H^{0}}^{2}\right)\right] \\
& -m_{\mu} \eta_{1} \eta_{2}^{*}\left[c_{\alpha}^{2} \mathcal{F}_{1}\left(m_{\chi_{2}}^{2}, m_{A^{0}}^{2}, m_{H^{0}}^{2}\right)+s_{\alpha}^{2} \mathcal{F}_{1}\left(m_{\chi_{1}}^{2}, m_{A^{0}}^{2}, m_{H^{0}}^{2}\right)\right] \\
& +m_{\mu} f_{1} f_{2}^{*}\left[s_{\delta}^{2} \mathcal{F}_{2}\left(m_{\kappa_{1}^{+}}^{2}, m_{N}^{2}\right)+c_{\delta}^{2} \mathcal{F}_{2}\left(m_{\kappa_{2}^{+}}^{2}, m_{N}^{2}\right)\right] .
\end{aligned}
$$



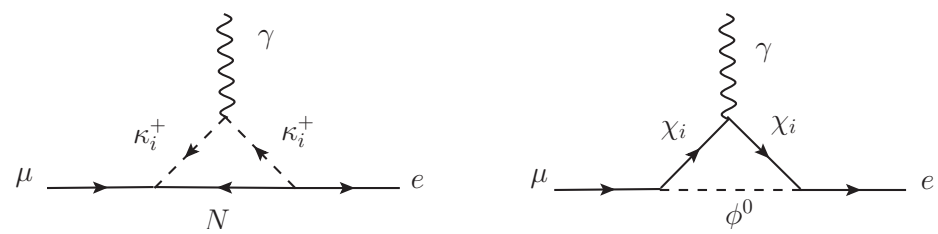

Figure 2. One-loop diagrams contributing to $\mu \rightarrow e \gamma$. In the right diagram $\phi^{0}$ denotes the two $Z_{2}$-odd neutral scalars $A^{0}$ and $H^{0}$.

The loop functions are presented in the appendix C. Note that, due to the equation (2.16), the couplings $\eta_{2}, \eta_{3}, f_{1}, f_{2}, f_{3}$ are related with $\eta_{1}$, hence, the only free Yukawa parameters entering in the expression for $\mathcal{B}(\mu \rightarrow e \gamma)$ are $\eta_{1}, \rho_{1}$, and $\rho_{2}$.

\section{Numerical results and discussion}

In order to illustrate the compatibility of the model with the experimental constraints, we consider the scenario without the extra annihilation channels discussed on section 2 . Furthermore, we set $H^{0}$ to be the DM candidate and assume a small mixing angle $\delta$ and the mass spectrum with the lightest charged scalar $\kappa_{1}^{ \pm}$mainly doublet. ${ }^{7}$

For the low mass regime and without lose of generality we assume $m_{\kappa_{1}^{+}}, m_{A^{0}}>100 \mathrm{GeV}$ and $|\delta| \lesssim 0.2$, which implies that the remaining $Z_{2}$-odd fields do not alter the DM phenomenology expected for the IDM in that regime. On the other hand, to quantitatively assess up to what extent the presence of the new fermion fields and $\kappa_{2}^{ \pm}$could affect the expected phenomenology in the high mass regime, through the opening of new (co)annihilation channels, we have calculated the DM relic density through micrOMEGAs [48] via FeynRules [49] and make a scan (to be described below) over the free parameters of the model. For this purpose, we have set $\lambda_{2}, \lambda_{S}$ and all the Yukawa-couplings to $10^{-2}$. The numerical result confirms the preliminary expectations: when $m_{\kappa_{2}^{+}} / m_{\kappa_{1}^{+}} \gtrsim 1.1,|\delta| \lesssim 0.2$ and $|\mu| / v \lesssim 10^{-1}$ the new (co-)annihilations channels compared with those present in the IDM do not play a significant role in the determination of DM relic density.

Regarding the electroweak precision test, we have performed a numerical analysis for the two DM mass regimes mentioned above. For the high mass regime, we have considered the following ranges for the free parameters:

$$
\begin{array}{ll}
500 \mathrm{GeV}<m_{H^{0}}<1 \mathrm{TeV} ; & m_{A^{0}}, m_{\kappa_{1}^{+}}=m_{H^{0}}+[0.1,10] \mathrm{GeV} ; \\
m_{\kappa_{2}^{+}}=m_{\kappa_{1}^{+}}+[0.1,1000] \mathrm{GeV} ; & m_{\chi_{1}}=m_{\kappa_{2}^{+}}+[0.1,1000] \mathrm{GeV} ; \\
m_{\chi_{2}}=m_{\chi_{1}}+[0.1,1000] \mathrm{GeV} ; & \delta, \alpha \in[-\pi / 2, \pi / 2] ; \quad \lambda_{L}, \lambda_{6} \in\left[10^{-3}, 1\right] .
\end{array}
$$

The scalar and fermion contributions to $S$ and $T$ are shown in figure 3 , where the constraints coming from the DM phenomenology mentioned above have been taken into account. The black, blue and green ellipses represent the experimental constraints at $68 \%$ CL, 95\% CL

\footnotetext{
${ }^{7}$ It is worth mentioning that when the lightest state $\kappa_{1}^{ \pm}$is mainly singlet, the relic density cannot be obtained without considering the coannihilation processes with $\kappa_{2}^{ \pm}$unless that $m_{\kappa_{1}^{ \pm}} \gtrsim 300 \mathrm{GeV}$ and $m_{H^{0}} \simeq m_{h} / 2$, in which case the relic density is independent of $m_{\kappa_{2}^{ \pm}} \cdot$
} 

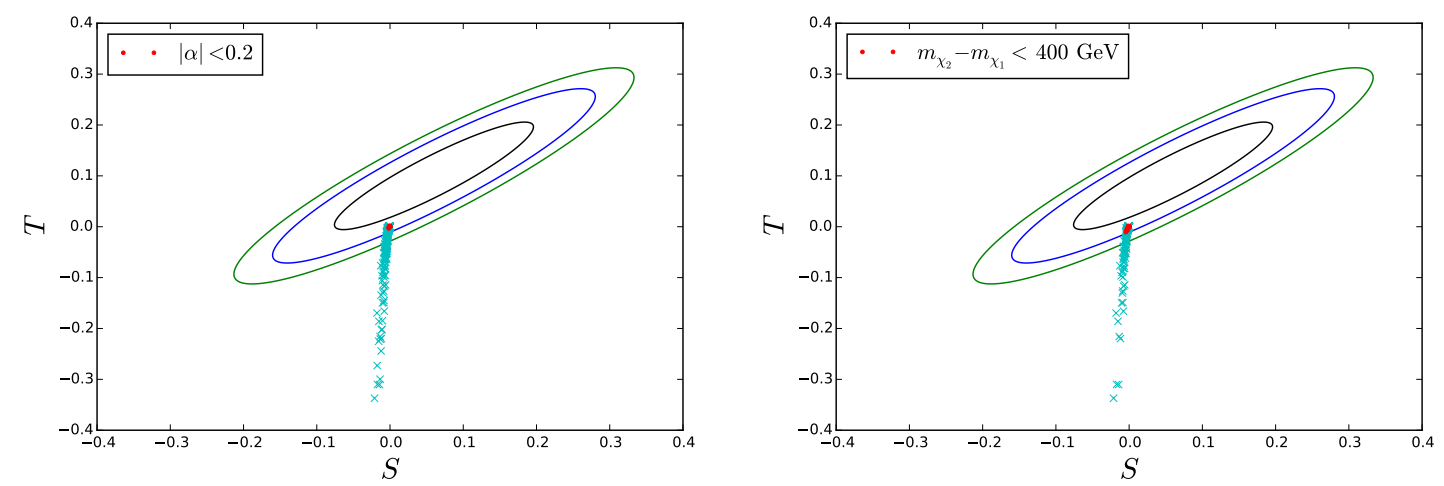

Figure 3. Contour plot for $Z_{2}$-odd scalar and fermion contributions to the EWPT parameters in the high mass regime. The left panel shows the $S, T$ contributions for any mass splitting $m_{\chi_{2}}-m_{\chi_{1}}$, while the right panel shows $S, T$ contributions for any value of the mixing angle $\alpha$.

and $99 \%$ CL, respectively [50]. ${ }^{8}$ It is worth to mention that contrary to the IDM, in our model the $S$ and $T$ parameters are not negligible in the high mass regime because the fermion contributions are already present. However, the constraints are easily satisfied for a small fermion mixing angle $|\alpha| \lesssim 0.2$ (red points in the left-panel). On the other hand, by allowing arbitrary values for the mixing angle, $\alpha$, the contributions to $S$ and $T$ are kept within the $2 \sigma$ level as long as $m_{\chi_{2}}-m_{\chi_{1}} \lesssim 400 \mathrm{GeV}$ (red points in the right-panel).

Regarding the low mass regime we have varied the free parameters as follows: $60 \mathrm{GeV}<$ $m_{H^{0}}<80 \mathrm{GeV}, 100 \mathrm{GeV}<m_{A^{0}}, m_{\kappa_{1}^{+}}<1000 \mathrm{GeV}, m_{\kappa_{1}^{+}}<m_{\kappa_{2}^{+}}<m_{\chi_{1}}<1000 \mathrm{GeV}$, and the same ranges in the eq. (4.1) for the mixing angles and scalar couplings. The fermion contributions to $S$ and $T$ are satisfied by imposing either $|\alpha| \lesssim 0.1$ or $m_{\chi_{2}}-m_{\chi_{1}} \lesssim 200 \mathrm{GeV}$. In this case, the scalar contributions are not kept within the $2 \sigma$ level by just imposing the DM phenomenology of the IDM. This occurs because in the low mass regime there is always a non-negligible mass splitting between the DM particle and the other scalars. Figure 4 shows the allowed values for the masses $m_{A^{0}}$ and $m_{\kappa_{1}^{+}}$that satisfy the $S, T$ parameters at $68 \%$ CL (red points), 95\% CL (green points) and 99\% CL (blue points) respectively. We have taken $|\alpha| \lesssim 0.1$ in order to suppress the fermion contribution. Note that if $m_{A^{0}}$ is increased, $m_{\kappa_{1}^{+}}$will have to be increased. However, from the unitary constraints given in eq. (2.6) an upper limit is obtained on the scalar masses, which leads to that they should be nearly degenerate at $800 \mathrm{GeV}$.

Concerning to the LFV constraints, we have focused on the current strongest bound, which is provided by $\mu \rightarrow e \gamma$ process. We have made a scan over the free parameters of the model for the CP-conserving scenario (the CP Dirac phase is fixed to zero) with a normal hierarchy and choosing $\lambda=-1$. For this purpose, we have varied the free parameters within the ranges given in eq. (4.1), in addition to $\eta_{1}, \rho_{1}, \rho_{2}, \in\left[10^{-4}, 1\right]$. The results are shown in figure 5 . All the points satisfy the current bound [51] and only a minority will be probed by future searches [52]. We have taken $|\alpha| \lesssim 0.1, m_{\kappa_{2}^{+}} / m_{\kappa_{1}^{+}} \gtrsim 1.1,|\delta| \lesssim 0.2$ and $|\mu| / v \lesssim 10^{-1}$

\footnotetext{
${ }^{8}$ The experimental deviations from the $\mathrm{SM}$ predictions in the $S$ and $T$ parameters for $m_{h}=126 \mathrm{GeV}$, $m_{t}=173 \mathrm{GeV}$ and $U=0$ are $S=0.06 \pm 0.09, T=0.10 \pm 0.07$ where the correlation factor between $S$ and $T$ is $0.91[50]$.
} 


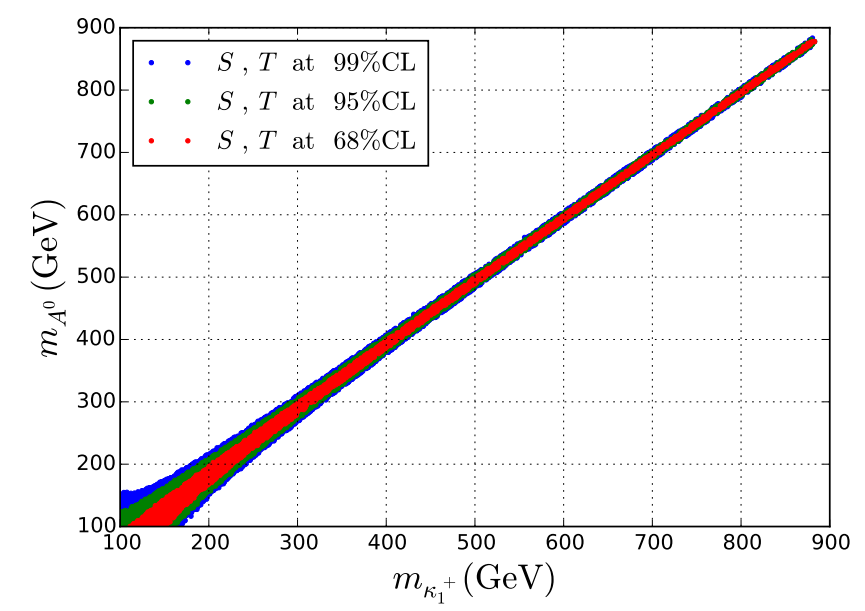

Figure 4. $S, T$ constraints on the masses $m_{A^{0}}$ and $m_{\kappa_{1}^{+}}$. We have taken $|\alpha|<0.1$ and $|\delta|<0.1$.

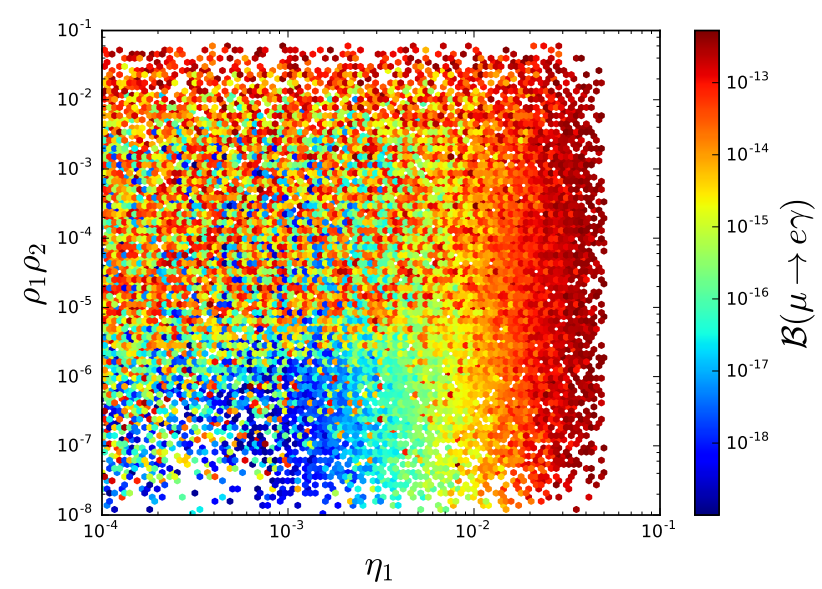

Figure 5. Region in the $\left(\eta_{1}, \rho_{1} \rho_{2}\right)$ plane for the high mass regime which is compatible with the current $\mu \rightarrow e \gamma$ constraint. Note that the correlation between $\rho_{1} \rho_{2}$ and $\eta$ can be spoiled by the interference between the two $\rho_{1} \rho_{2}$ contributions and/or by the $\rho_{1}^{*} \eta_{2}^{*}$ and $\rho_{2} \eta_{1}$ contributions (see eqs. (25) and (26)), since it is possible to obtain low values of $\mathcal{B}(\mu \rightarrow e \gamma$ ) (color code) with relative large values of $\rho_{1} \rho_{2} \sim 10^{-2}$. For a inverted hierarchy and $\lambda=-1$ the numerical results are similiar to those for the normal hierarchy: $\rho_{1} \rho_{2} \leq 0.08$ and $\eta_{1} \leq 0.3$ for the current bound.

in order to satisfy the oblique parameters and preserve the DM phenomenology expected for the IDM. Note that the $\mathcal{B}(\mu \rightarrow e \gamma)$ limit can be easily satisfied imposing $\rho_{1} \rho_{2} \lesssim 4 \times 10^{-2}$ and $\eta_{1} \lesssim 5 \times 10^{-2}$. On the other hand, for the low mass regime we obtain similar results to those in the high mass regime. Remember that, in order to satisfy the oblique parameter we need to impose small mixing angles as well as a nearly degenerate masses between $A^{0}$ and $\kappa_{1}^{+}$.

Finally, we turn the discussion to collider searches. The high-mass region of the IDM is quite difficult to probe at the LHC. However, the low mass region can be probed by searching for dilepton plus missing transverse energy signal [53-55] and trilepton plus 
missing transverse energy signal $[56,57]$ with a sensitivity in the parameter region with $\kappa_{1}^{+}, A^{0} 100-180 \mathrm{GeV}$. A similar sensitivity could be expected for $\kappa_{2}^{+}$.

Concerning VL fermions, the searches performed at LEPII impose a limit of $m_{\chi_{1}}>$ $100 \mathrm{GeV}$ [58]. At the LHC, the larger exclusion for VL fermion is expected for large mass splittings, $100 \%$ branching ratios to electron or muons, and higher fermions $\mathrm{SU}(2)_{L}$ representations. In our case, it corresponds to a higgsino-like VL fermion production without final state taus. For example, if a higgsino-like charged fermion is the next to lightest $Z_{2}$-odd particle and choosing the Yukawa-couplings such that

$$
\max \left(\rho_{1}, \rho_{2}\right) \gg \min \left(\rho_{1}, \rho_{2}\right), \rho_{3}, \eta_{i},
$$

we have a dilepton plus missing transverse energy signal from

$$
p p \rightarrow \chi_{1}^{+} \chi_{1}^{-} \rightarrow l^{+} l^{-} H^{0} H^{0}, \quad \quad l^{ \pm}=e^{ \pm}, \mu^{ \pm} .
$$

Since the cuts for this kind of signal at the LHC (in both ATLAS and CMS) do not depend in angular distributions between the final states, the corresponding excluded cross sections are insensitive to the spin of the produced particles. Currently, they are interpreted in terms of slepton pair production. A recast of the excluded cross section for slepton pair production $p p \rightarrow \tilde{l}^{+} \tilde{l}^{-} \rightarrow l^{+} l^{-} \tilde{\chi}_{1}^{0} \tilde{\chi}_{1}^{0}$, studied in ref. [59], ${ }^{9}$ allows to exclude higgsino-like charged fermions up to $510 \mathrm{GeV}[15]$.

Conversely, in the case of $\chi_{1}^{ \pm}$nearly degenerate with $H^{0}$ (compressed spectra), the bounds on $m_{\chi_{1}}$ are $\sim 100 \mathrm{GeV}$ for $\Delta m=m_{\chi_{1}}-m_{H^{0}}<50 \mathrm{GeV}$ [60]. ${ }^{10}$ If, in addition, the Yukawa-couplings are such that

$$
\rho_{3} \gg \rho_{1}, \rho_{2}, \eta_{i}
$$

then $\mathcal{B}\left(\chi^{ \pm} \rightarrow \tau H^{0}\right) \approx 1$, and the exclusion limits are worse due to the larger $\tau$ misidentification rates. Recently, an extended analysis of the LHC Run-I data have been presented by ATLAS [61] with new searches for compressed spectra and final state taus. In particular, by using multivariate analysis techniques, the $95 \%$ excluded cross section for $p p \rightarrow \tilde{\tau}_{R, L}^{+} \tilde{\tau}_{R, L}^{-} \rightarrow \tau^{+} \tau^{-} \tilde{\chi}_{1}^{0} \tilde{\chi}_{1}^{0}$ is given for several neutralino masses. As expected, and in contrast to the selectron and smuon pair production, there is no sensitivity to left- or right-stau pair production. By using the same strategy than in [62], we focus in the excluded cross section plot presented in figure 12 of ref. [61] for a DM particle of $60 \mathrm{GeV}$, since it is a representative value in the case of the IDM to account for the proper relic density. Because of the larger cross section for pair produced higgsinos decaying into two taus plus missing transverse energy, we are able to exclude higgsino-like charged fermions in the range $115<m_{\chi_{1}^{+}} / \mathrm{GeV}<180$ by using the theoretical cross section calculated to next-to-leading order in [15].

Another attempt to circumvent both problems have been made recently in ref. [60] of the CMS collaboration, by implementing the vector boson fusion topology to pair produce electroweakinos [63]. There, supersymmetric models with bino-like $\widetilde{\chi}_{1}^{0}$ and wino-like $\widetilde{\chi}_{2}^{0}$ and

\footnotetext{
${ }^{9}$ Where the lightest neutralino, $\tilde{\chi}_{1}^{0}$, is the dark matter candidate.

${ }^{10} \mathrm{In}$ our case, the low mass region of the IDM with $m_{H^{0}}=70 \mathrm{GeV}$ combined with the LEPII constraint on VL fermions, imply that $\Delta m>30 \mathrm{GeV}$.
} 
$\widetilde{\chi}_{1}^{ \pm}$are considered in the presence of a light stau. Assuming $\mathcal{B}\left(\widetilde{\chi}_{1}^{ \pm} \rightarrow \nu \widetilde{\tau}^{ \pm} \rightarrow \nu \tau^{ \pm} \widetilde{\chi}_{1}^{0}\right)=1$ and $\mathcal{B}\left(\widetilde{\chi}_{2}^{0} \rightarrow \tau^{ \pm} \widetilde{\tau}^{\mp} \rightarrow \tau^{ \pm} \tau^{\mp} \widetilde{\chi}_{1}^{0}\right)=1$, they are able to find some supersymmetric scenarios where the LEPII constraint can be improved. We could expect that a similar analysis for the higgsino-like charged VL fermion may allow to close the previous gap until around $115 \mathrm{GeV}$. A detailed recast of this CMS analysis, will be done elsewhere. In summary, we expect an exclusion for the higgsino-like charged VL fermions of the model around $180 \mathrm{GeV}$. On the other hand, searches in the di-tau plus missing transverse energy signature have been studied in ref. [64]. There, it was shown that the high luminosity LHC of $3000 \mathrm{fb}^{-1}$ can exclude $\mathrm{SU}(2)_{L}$-singlet charged VL fermion up to $m_{\chi_{1}} \sim 450 \mathrm{GeV}$.

\section{Conclusions}

We have considered an extension of the Zee model which involves two vector-like leptons, a doublet and a singlet of $\mathrm{SU}(2)_{L}$ and the imposition of an exact $Z_{2}$ symmetry. This symmetry, under which all the non-Standard Model fields are odd, avoids tree-level Higgsmediated flavor changing neutral currents and ensures the stability of the lightest neutral component inside the second scalar doublet and, therefore, allowing to have a viable dark matter candidate. We have shown that under some conditions the well-known DM phenomenology of the IDM is recovered. As in the Zee model, neutrino masses are generated at one loop, leading to either a normal mass hierarchy or a inverted mass hierarchy. However, due to the flavor structure of the neutrino mass matrix, one neutrino remains massless. Moreover, such a flavor structure always allows to reproduce the correct neutrino oscillation parameters and to have only four free Yukawa-couplings (of a total of nine), which can be constrained using the $\mu \rightarrow e \gamma$ lepton flavor violation process. In particular, we have found that $\rho_{1} \rho_{2} \lesssim 10^{-2}$ and $\eta_{1} \lesssim 10^{-2}$ in order to fulfill that constraint. On the other hand, the oblique parameters impose $|\alpha| \lesssim 0.2$ and $m_{\chi_{2}}-m_{\chi_{1}} \lesssim 400 \mathrm{GeV}$ for the high mass regime while $|\alpha| \lesssim 0.1$ and $m_{\chi_{2}}-m_{\chi_{1}} \lesssim 200 \mathrm{GeV}$ for the low mass regime. Finally, we argued that in general, the collider limits for vector-like leptons are not so far from the limit imposed by LEPII.

\section{Acknowledgments}

We are very gratefully to Federico von der Pahlen for illuminating discussions. D. R. and O. Z. are supported by UdeA through the grants Sostenibilidad-GFIF, CODI-2014361 and CODI-IN650CE, and COLCIENCIAS through the grants numbers 111-556-934918 and 111-565-842691. D. P. and R. L. are supported by COLCIENCIAS. R. L. acknowledges the hospitality of Universidade Federal do ABC in the final stage of this work.

\section{A Free parameters}

Some of the scalar potential parameters can be written in terms of physical scalar masses using the relations in (2.2) and (2.5):

$$
\begin{array}{rlrl}
\frac{1}{2} v^{2} \lambda_{3} & =m_{\kappa_{1}^{+}}^{2} \cos ^{2} \delta+m_{\kappa_{2}^{+}}^{2} \sin ^{2} \delta-m_{H^{0}}^{2}+v^{2} \lambda_{L}, & \mu_{S}^{2}=m_{\kappa_{1}^{+}}^{2} \sin ^{2} \delta+m_{\kappa_{2}^{+}}^{2} \cos ^{2} \delta-\frac{1}{2} v^{2} \lambda_{6}, \\
v^{2} \lambda_{5} & =m_{H^{0}}^{2}-m_{A^{0}}^{2}, \quad \lambda_{4}=2 \lambda_{L}-\lambda_{3}-\lambda_{5}, \quad \mu_{2}^{2}=m_{H^{0}}^{2}-\lambda_{L} v^{2} .
\end{array}
$$




\section{B ST formulae}

Here, we present the analytical loop functions used for the analysis of the $S$ and $T$ parameters,

$$
\begin{aligned}
\Theta_{S}\left(m_{1}, m_{2}\right)= & \frac{2}{9}+\frac{\left(m_{1}^{2}+m_{2}^{2}\right)\left(m_{1}^{4}-4 m_{1}^{2} m_{2}^{2}+m_{2}^{4}\right)+6 m_{1}^{3} m_{2}^{3}}{6\left(m_{1}^{2}-m_{2}^{2}\right)^{3}} \log \left(\frac{m_{1}^{2}}{m_{2}^{2}}\right) \\
& +\frac{4 m_{1}^{2} m_{2}^{2}-3 m_{1} m_{2}\left(m_{1}^{2}+m_{2}^{2}\right)}{6\left(m_{1}^{2}-m_{2}^{2}\right)^{2}}, \\
\Theta_{T}\left(m_{1}, m_{2}\right)= & \frac{m_{1}^{2}+m_{2}^{2}}{4}-\frac{\left(m_{1}^{4}-2 m_{1} m_{2}\left(m_{1}^{2}+m_{2}^{2}\right)+m_{2}^{4}\right)}{4\left(m_{1}^{2}-m_{2}^{2}\right)} \log \left(\frac{m_{2}^{2}}{m_{1}^{2}}\right), \\
\Theta\left(p^{2} ; m_{1}, m_{2}\right)= & \int_{0}^{1} d x\left[(2 x-1)\left(m_{1}^{2}-m_{2}^{2}\right)+(2 x-1)^{2} p^{2}\right] \\
\Theta\left(0 ; m_{1}, m_{2}\right)= & \frac{m_{1}^{2}+m_{2}^{2}}{2}-\frac{m_{1}^{2} m_{2}^{2}}{m_{1}^{2}-m_{2}^{2}} \ln \left(\frac{m_{1}^{2}}{m_{2}^{2}}\right) .
\end{aligned}
$$

\section{Loop function in the $\mu \rightarrow e \gamma$}

Here, we present the analytical loop functions used for the analysis of the $\mu \rightarrow e \gamma$ constraint,

$$
\begin{aligned}
\mathcal{G}_{1}\left(m_{a}^{2}, m_{b}^{2}, m_{c}^{2}\right) & =\frac{1}{m_{b}^{2}} G\left(\frac{m_{a}^{2}}{m_{b}^{2}}\right)-\frac{1}{m_{c}^{2}} G\left(\frac{m_{a}^{2}}{m_{c}^{2}}\right), \\
\mathcal{F}_{1}\left(m_{a}^{2}, m_{b}^{2}, m_{c}^{2}\right) & =\frac{1}{2 m_{a}^{2}}\left[F\left(\frac{m_{b}^{2}}{m_{a}^{2}}\right)+F\left(\frac{m_{c}^{2}}{m_{a}^{2}}\right)\right], \\
\mathcal{F}_{2}\left(m_{a}^{2}, m_{b}^{2}\right) & =\mathcal{F}_{1}\left(m_{a}^{2}, m_{b}^{2}, m_{b}^{2}\right),
\end{aligned}
$$

where

$$
F(x)=\frac{2 x^{3}+3 x^{2}-6 x+1-6 x^{2} \log (x)}{6(x-1)^{4}}, \quad G(x)=\frac{x^{2}-4 x+3+2 \log (x)}{2(x-1)^{3}} .
$$

Open Access. This article is distributed under the terms of the Creative Commons Attribution License (CC-BY 4.0), which permits any use, distribution and reproduction in any medium, provided the original author(s) and source are credited.

\section{References}

[1] E. Ma, Pathways to naturally small neutrino masses, Phys. Rev. Lett. 81 (1998) 1171 [hep-ph/9805219] [INSPIRE].

[2] F. Bonnet, M. Hirsch, T. Ota and W. Winter, Systematic study of the D=5 Weinberg operator at one-loop order, JHEP 07 (2012) 153 [arXiv: 1204.5862] [INSPIRE].

[3] D. Aristizabal Sierra, A. Degee, L. Dorame and M. Hirsch, Systematic classification of two-loop realizations of the Weinberg operator, JHEP 03 (2015) 040 [arXiv:1411.7038] [INSPIRE]. 
[4] S.S.C. Law and K.L. McDonald, A class of inert $N$-tuplet models with radiative neutrino mass and dark matter, JHEP 09 (2013) 092 [arXiv: 1305.6467] [INSPIRE].

[5] D. Restrepo, O. Zapata and C.E. Yaguna, Models with radiative neutrino masses and viable dark matter candidates, JHEP 11 (2013) 011 [arXiv:1308.3655] [INSPIRE].

[6] G.C. Branco, P.M. Ferreira, L. Lavoura, M.N. Rebelo, M. Sher and J.P. Silva, Theory and phenomenology of two-Higgs-doublet models, Phys. Rept. 516 (2012) 1 [arXiv:1106.0034] [INSPIRE].

[7] A. Zee, A theory of lepton number violation, neutrino Majorana mass and oscillation, Phys. Lett. B 93 (1980) 389 [Erratum ibid. B 95 (1980) 461] [INSPIRE].

[8] L. Wolfenstein, A theoretical pattern for neutrino oscillations, Nucl. Phys. B 175 (1980) 93 [INSPIRE].

[9] X.-G. He, Is the Zee model neutrino mass matrix ruled out?, Eur. Phys. J. C 34 (2004) 371 [hep-ph/0307172] [INSPIRE].

[10] N.G. Deshpande and E. Ma, Pattern of symmetry breaking with two Higgs doublets, Phys. Rev. D 18 (1978) 2574 [INSPIRE].

[11] E. Ma, Verifiable radiative seesaw mechanism of neutrino mass and dark matter, Phys. Rev. D 73 (2006) 077301 [hep-ph/0601225] [INSPIRE].

[12] E. Ma and D. Suematsu, Fermion triplet dark matter and radiative neutrino mass, Mod. Phys. Lett. A 24 (2009) 583 [arXiv:0809.0942] [InSPIRE].

[13] W. Chao, Dark matter, LFV and neutrino magnetic moment in the radiative seesaw model with fermion triplet, Int. J. Mod. Phys. A 30 (2015) 1550007 [arXiv:1202.6394] [inSPIRE].

[14] S. Fraser, E. Ma and O. Popov, Scotogenic inverse seesaw model of neutrino mass, Phys. Lett. B 737 (2014) 280 [arXiv:1408.4785] [InSPIRE].

[15] D. Restrepo et al., Radiative neutrino masses in the singlet-doublet fermion dark matter model with scalar singlets, Phys. Rev. D 92 (2015) 013005 [arXiv: 1504.07892] [INSPIRE].

[16] H. Okada and Y. Orikasa, Two-loop neutrino model with exotic leptons, Phys. Rev. D 93 (2016) 013008 [arXiv: 1509.04068] [INSPIRE].

[17] S. Davidson and H.E. Haber, Basis-independent methods for the two-Higgs-doublet model, Phys. Rev. D 72 (2005) 035004 [Erratum ibid. D 72 (2005) 099902] [hep-ph/0504050] [INSPIRE].

[18] D. Aristizabal Sierra and D. Restrepo, Leptonic charged Higgs decays in the Zee model, JHEP 08 (2006) 036 [hep-ph/0604012] [INSPIRE].

[19] X.-G. He and S.K. Majee, Implications of recent data on neutrino mixing and lepton flavour violating decays for the Zee model, JHEP 03 (2012) 023 [arXiv:1111.2293] [INSPIRE].

[20] T. Fukuyama, H. Sugiyama and K. Tsumura, Phenomenology in the Zee model with the $A_{4}$ symmetry, Phys. Rev. D 83 (2011) 056016 [arXiv: 1012.4886] [INSPIRE].

[21] G. Blankenburg, J. Ellis and G. Isidori, Flavour-changing decays of a $125 \mathrm{GeV}$ Higgs-like particle, Phys. Lett. B 712 (2012) 386 [arXiv:1202.5704] [INSPIRE].

[22] R. Harnik, J. Kopp and J. Zupan, Flavor Violating Higgs Decays, JHEP 03 (2013) 026 [arXiv:1209.1397] [INSPIRE]. 
[23] R. Dermisek, E. Lunghi and S. Shin, Two Higgs doublet model with vectorlike leptons and contributions to $p p \rightarrow W W$ and $H \rightarrow W W$, JHEP 02 (2016) 119 [arXiv:1509.04292] [INSPIRE].

[24] F.J. Botella, G.C. Branco, M. Nebot and M.N. Rebelo, Flavour changing Higgs couplings in a class of two Higgs doublet models, arXiv:1508.05101 [INSPIRE].

[25] W. Altmannshofer, J. Brod and M. Schmaltz, Experimental constraints on the coupling of the Higgs boson to electrons, JHEP 05 (2015) 125 [arXiv: 1503.04830] [INSPIRE].

[26] S. Kanemura, T. Shindou and H. Sugiyama, R-parity conserving supersymmetric extension of the Zee model, Phys. Rev. D 92 (2015) 115001 [arXiv:1508.05616] [InSPIRE].

[27] R. Barbieri, L.J. Hall and V.S. Rychkov, Improved naturalness with a heavy Higgs: an alternative road to LHC physics, Phys. Rev. D 74 (2006) 015007 [hep-ph/0603188] [INSPIRE].

[28] L. Lopez Honorez, E. Nezri, J.F. Oliver and M.H.G. Tytgat, The inert doublet model: an archetype for dark matter, JCAP 02 (2007) 028 [hep-ph/0612275] [INSPIRE].

[29] S. Kanemura, T. Kasai, G.-L. Lin, Y. Okada, J.-J. Tseng and C.P. Yuan, Phenomenology of Higgs bosons in the Zee model, Phys. Rev. D 64 (2001) 053007 [hep-ph/0011357] [InSPIRE].

[30] M. Gustafsson, E. Lundstrom, L. Bergstrom and J. Edsjo, Significant $\gamma$ lines from inert Higgs dark matter, Phys. Rev. Lett. 99 (2007) 041301 [astro-ph/0703512] [INSPIRE].

[31] C. Garcia-Cely, M. Gustafsson and A. Ibarra, Probing the inert doublet dark matter model with Cherenkov telescopes, JCAP 02 (2016) 043 [arXiv: 1512.02801] [INSPIRE].

[32] T. Abe and R. Sato, Quantum corrections to the spin-independent cross section of the inert doublet dark matter, JHEP 03 (2015) 109 [arXiv: 1501.04161] [INSPIRE].

[33] A. Goudelis, B. Herrmann and O. Stål, Dark matter in the inert doublet model after the discovery of a Higgs-like boson at the LHC, JHEP 09 (2013) 106 [arXiv:1303.3010] [INSPIRE].

[34] Z. Maki, M. Nakagawa and S. Sakata, Remarks on the unified model of elementary particles, Prog. Theor. Phys. 28 (1962) 870 [inSPIRE].

[35] Particle Data Group collaboration, K.A. Olive et al., Review of particle physics, Chin. Phys. C 38 (2014) 090001 [INSPIRE].

[36] L. Lopez Honorez and C.E. Yaguna, The inert doublet model of dark matter revisited, JHEP 09 (2010) 046 [arXiv: 1003.3125] [INSPIRE].

[37] L. Lopez Honorez and C.E. Yaguna, A new viable region of the inert doublet model, JCAP 01 (2011) 002 [arXiv: 1011.1411] [INSPIRE].

[38] C. Garcia-Cely and A. Ibarra, Novel $\gamma$-ray spectral features in the inert doublet model, JCAP 09 (2013) 025 [arXiv: 1306.4681] [INSPIRE].

[39] A. Arhrib, Y.-L.S. Tsai, Q. Yuan and T.-C. Yuan, An updated analysis of inert Higgs doublet model in light of the recent results from LUX, PLANCK, AMS-02 and LHC, JCAP 06 (2014) 030 [arXiv:1310.0358] [INSPIRE].

[40] K. Griest and D. Seckel, Three exceptions in the calculation of relic abundances, Phys. Rev. D 43 (1991) 3191 [INSPIRE]. 
[41] S. Profumo and A. Provenza, Increasing the neutralino relic abundance with slepton coannihilations: consequences for indirect dark matter detection, JCAP 12 (2006) 019 [hep-ph/0609290] [INSPIRE].

[42] M. Klasen, C.E. Yaguna, J.D. Ruiz-Alvarez, D. Restrepo and O. Zapata, Scalar dark matter and fermion coannihilations in the radiative seesaw model, JCAP 04 (2013) 044 [arXiv: 1302.5298] [INSPIRE].

[43] M.E. Peskin and T. Takeuchi, Estimation of oblique electroweak corrections, Phys. Rev. D 46 (1992) 381 [INSPIRE].

[44] G. Cynolter and E. Lendvai, Electroweak precision constraints on vector-like fermions, Eur. Phys. J. C 58 (2008) 463 [arXiv:0804.4080] [inSPIRE].

[45] G. Cynolter, E. Lendvai and G. Pocsik, $S$ and T parameters in the Fermion condensate model, Mod. Phys. Lett. A 24 (2009) 2331 [arXiv:0904.1090] [INSPIRE].

[46] H. Okada, T. Toma and K. Yagyu, Inert extension of the Zee-Babu model, Phys. Rev. D 90 (2014) 095005 [arXiv: 1408.0961] [INSPIRE].

[47] R. Barbieri, A. Pomarol, R. Rattazzi and A. Strumia, Electroweak symmetry breaking after LEP-1 and LEP-2, Nucl. Phys. B 703 (2004) 127 [hep-ph/0405040] [INSPIRE].

[48] G. Bélanger, F. Boudjema, A. Pukhov and A. Semenov, MicrOMEGAs_3: a program for calculating dark matter observables, Comput. Phys. Commun. 185 (2014) 960 [arXiv: 1305.0237] [INSPIRE].

[49] A. Alloul, N.D. Christensen, C. Degrande, C. Duhr and B. Fuks, FeynRules 2.0 - A complete toolbox for tree-level phenomenology, Comput. Phys. Commun. 185 (2014) 2250 [arXiv: 1310.1921] [INSPIRE].

[50] GFitTer Group collaboration, M. Baak et al., The global electroweak fit at NNLO and prospects for the LHC and ILC, Eur. Phys. J. C 74 (2014) 3046 [arXiv:1407.3792] [INSPIRE].

[51] MEG collaboration, J. Adam et al., New constraint on the existence of the $\mu^{+} \rightarrow e^{+} \gamma$ decay, Phys. Rev. Lett. 110 (2013) 201801 [arXiv:1303.0754] [INSPIRE].

[52] A.M. Baldini et al., MEG upgrade proposal, arXiv:1301.7225 [inSPIRE].

[53] E. Dolle, X. Miao, S. Su and B. Thomas, Dilepton signals in the inert doublet model, Phys. Rev. D 81 (2010) 035003 [arXiv: 0909.3094] [InSPIRE].

[54] G. Bélanger et al., Dilepton constraints in the inert doublet model from Run 1 of the LHC, Phys. Rev. D 91 (2015) 115011 [arXiv:1503.07367] [INSPIRE].

[55] A. Ilnicka, M. Krawczyk and T. Robens, The inert doublet model in the light of LHC and astrophysical data - An update, Phys. Rev. D 93 (2016) 055026 [arXiv:1508.01671] [INSPIRE].

[56] X. Miao, S. Su and B. Thomas, Trilepton signals in the inert doublet model, Phys. Rev. D 82 (2010) 035009 [arXiv: 1005.0090] [InSPIRE].

[57] T. Abe, R. Kitano and R. Sato, Discrimination of dark matter models in future experiments, Phys. Rev. D 91 (2015) 095004 [arXiv:1411.1335] [inSPIRE].

[58] L3 collaboration, P. Achard et al., Search for heavy neutral and charged leptons in $e^{+} e^{-}$ annihilation at LEP, Phys. Lett. B 517 (2001) 75 [hep-ex/0107015] [INSPIRE]. 
[59] ATLAS collaboration, Search for direct production of charginos, neutralinos and sleptons in final states with two leptons and missing transverse momentum in pp collisions at $\sqrt{s}=8 \mathrm{TeV}$ with the ATLAS detector, JHEP 05 (2014) 071 [arXiv:1403.5294] [INSPIRE].

[60] CMS collaboration, Search for supersymmetry in the vector-boson fusion topology in proton-proton collisions at $\sqrt{s}=8 \mathrm{TeV}$, JHEP 11 (2015) 189 [arXiv:1508.07628] [INSPIRE].

[61] ATLAS collaboration, Search for the electroweak production of supersymmetric particles in $\sqrt{s}=8$ TeV pp collisions with the ATLAS detector, Phys. Rev. D 93 (2016) 052002 [arXiv: 1509.07152] [INSPIRE].

[62] F. von der Pahlen, G. Palacio, D. Restrepo and O. Zapata, Radiative Type III Seesaw Model and its collider phenomenology, in preparation.

[63] B. Dutta et al., Vector boson fusion processes as a probe of supersymmetric electroweak sectors at the LHC, Phys. Rev. D 87 (2013) 035029 [arXiv:1210.0964] [INSPIRE].

[64] Z.-H. Yu, X.-J. Bi, Q.-S. Yan and P.-F. Yin, $\tau$ portal dark matter models at the LHC, Phys. Rev. D 91 (2015) 035008 [arXiv: 1410.3347] [INSPIRE]. 